\title{
La « simplexité » des contes
}

\section{Can the concept of « simplexity » help us to rethink the French literary fairy tales and Perrault's poetic project?}

\author{
Patricia Eichel-Lojkine
}

Université du Maine

\begin{abstract}
The tale is not just a linear, sober narrative, but a clever combination of a diegesis of a certain type, a network of images and an implicit philosophy. This is where it differs from both the short story and the fable. As a literary genre, it is less of a « simple form » (André Jolles) and more of a "simplex form » (Alain Berthoz). In this context, how to address the question of the simple style in the literary genre of the fairy tale - a genre which leans towards complexity and sophistication, more than simplicity? We will suggest that the literary ideal of simplicity advocated by Perrault, best exemplified in The Fairies, is constructed against the gallant aesthetic which was the source of French fairy tales.
\end{abstract}

Keywords: André Jolles, Alain Berthoz, Charles Perrault, Mme d'Aulnoy, Book of tales, Book of fables, Naïvety, Simplification, The Fairies

Les contes sont des récits à la structure particulière, qui se transmettent à la fois comme forme verbale et écrite, qui peuvent recevoir des degrés d'élaboration et de sophistication très variables, et qui émergent en tout cas sur la scène littéraire européenne précisément au XVI ${ }^{\mathrm{e}}$ siècle. Nous nous demanderons ici si la simplicité est une "entrée » pertinente, une notion opératoire, pour rendre compte de leur poétique. En quoi la notion de simplicité peut-elle aider à théoriser, à modéliser les premiers contes de fées littéraires, c'est-à-dire les contes merveilleux italiens et français des $\mathrm{XVI}^{\mathrm{e}}$ et $\mathrm{XVII}^{\mathrm{e}}$ siècles (à supposer qu'ils puissent se regrouper dans un ensemble cohérent) ?

Ces textes ne répondent pas à une norme, ne se plient pas à une codification esthétique comme le fait la fable ou les formes définies par la poétique aristotélicienne. Ils recyclent des matériaux de toutes origines : contes antiques ${ }^{1}$; contes

\footnotetext{
${ }^{1}$ Car l'Antiquité connaît le conte comme le prouve la fabella d'Amour et Psyché enchâssée dans les Métamorphoses d'Apulée (Sermain, 2010a : 14).
} 
de tradition populaire (dont on sait peu de choses); fables d'origine orientale (Berlioz \& Polo de Beaulieu, 2008 : 168 ; Tolan, 1993 : 139sq.), comme les Fables de Bidpaï (ou fables du Kalila et Dimna d'Ibn Al-Muqaffà) passées par différents intermédiaires². Ainsi, par des voies variées, le Moyen Âge absorbe des histoires divertissantes ou des fables à visée didactique et politique et en nourrit sa littérature vernaculaire. Des contes trouvent une terre d'accueil dans certains exempla ${ }^{3}$, dans des lais ${ }^{4}$, dans des romans de chevalerie ${ }^{5}$.

Pour Jean-Paul Sermain, « le conte est comme une fable qui a mal tourné, c'est-à-dire qui s'affranchit de son ancrage rhétorique et pédagogique (dans le double sens qu'elle fait l'objet d'un apprentissage et qu'elle transmet des leçons utiles) » (Sermain, 2010a : 14). Si l'on voit le conte s'affranchir de l'ancrage pédagogique, c'est sans doute par contamination avec la tradition de la novellistica qui s'épanouit en milieu urbain ${ }^{6}$, à Florence, puis à Venise où Le Piacevoli Notti de Straparola font figure de bestseller à partir des années 1550-1553 (dates de parution de ses deux volumes).

Des transformations littéraires résultent de cette émancipation de plus en plus prononcée par rapport à la fable, que résume ainsi Sermain :

Le récit y garde sa netteté, son autonomie : tous les éléments narratifs sont subordonnés à la formation et à la perception de l'histoire, et l'affranchissement du rhétorique et du pédagogique conduit à intensifier et à exploiter les qualités propres du récit - complexité, surprise, effet comique ou pathétique, extraordinaire (Sermain, 2010a : 14) ${ }^{7}$.

La différenciation entre fables (simples ?) et contes (complexes ?) est particulièrement intéressante concernant Perrault qui traduisit en français en 1699 les fables latines de Gabriele Faerne (Fabulae centum, Rome, P. Manuzio, 1563). Elle prend sens aussi par rapport à la tradition initiée par Straparola à Venise si l'on songe aux Cento Favole Morali de Giovan Mario Verdizzotti, un poète et un illustrateur de talent (Venise, G. Zileti, 1570) ${ }^{8}$. Considérons le vieux bûcheron portraituré dans un paysage assez réaliste dans la fameuse fable du «Vieillard et la Mort » (fig. 1) : on pourrait trouver personnage et paysage semblables dans les novellas ou les contes du temps. Les interactions entre fables, novellas et contes ne doivent donc pas être négligées.

\footnotetext{
${ }^{2}$ Le Syntipas grec, la Disciplina clericalis de Pierre Alphonse, le Directorium vitae humanae de Jean de Capoue ou le Livre des Sept Sages de Rome...

${ }^{3}$ La Scala coeli de Jean de Gobi junior (XIV ${ }^{\mathrm{e}}$ siècle).

${ }^{4}$ Marie de France (XII ${ }^{\mathrm{e}}$ siècle).

${ }^{5}$ Comme le Perceforest (anonyme, XIV ${ }^{\mathrm{e}}$ siècle).

${ }^{6}$ Pour un résumé rapide des rapports entre contes et nouvelles à partir du XVI ${ }^{\mathrm{e}}$ siècle, voir Jolles (1970 : 180-182).

${ }^{7}$ Nous soulignons.

${ }^{8}$ Cf. Corradi (2013 : 73-82).
} 


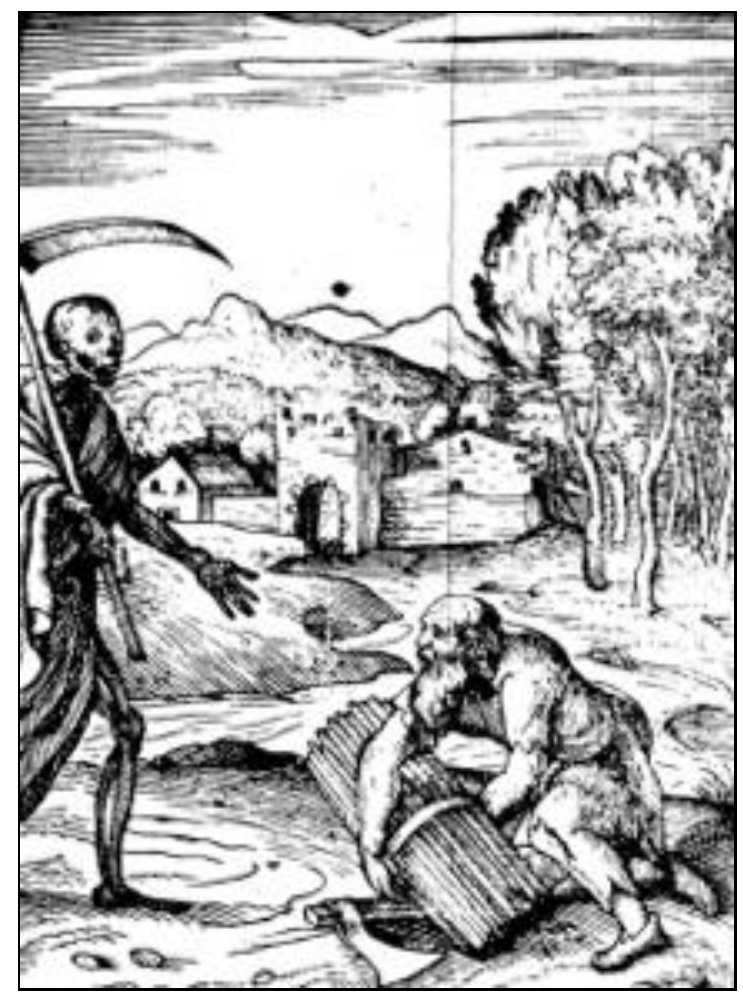

Fig. 1. «D’un vecchio e la morte »

Faut-il néanmois en déduire que les contes seraient plutôt du côté de la complexité, avec des récits qui dessinent des parcours perturbés, qui peuvent être amplifiés selon l'inspiration et la copia du conteur, et qui ouvrent en outre sur un horizon herméneutique incertain ? Car chez Straparola, la récitation de chaque «favola» ne se conclut pas par une moralité, mais est suivie d'une énigme dont le déchiffrement est proposé à l'assemblée. Les moralités sont une invention des contes de fées français qui les empruntent au modèle jalousé des Fables de La Fontaine, mais chez Perrault, un narrateur malicieux s'amuse à les dédoubler, voire à les tripler, de sorte qu'elles ne se superposent qu'imparfaitement et même parfois se contredisent ${ }^{9}$. Faut-il opposer les contes aux fables qui seraient,

${ }^{9}$ Pour prendre l'exemple des Fées, que nous analyserons plus loin, la première moralité («[...] les douces paroles [...] sont d'un plus grand prix ») tire l'image des fleurs et des perles sortant de la bouche du côté de l'allégorisation du «doux parler», en accord avec La Fontaine («Le Cygne et le cuisinier», III, 12) et Mlle Lhéritier (Les Enchantements de l'éloquence) ; la seconde se conforme au conte d'avertissement prônant la conduite serviable («L'honnêteté [...] tôt ou tard a sa récompense »). 
elles, plutôt du côté de la simplicité, avec leur culture de la leçon, leur modèle rhétorique et oratoire (reposant sur l'échange de discours entre les personnages), leur vision pragmatique et leur traditionnel emploi pédagogique ?

\section{LE CONCEPT DE «SIMPLEXITE» PEUT-IL S'APPLIQUER AUX CONTES ?}

Les contes sont des formes ductiles, plastiques, inscrites dans un processus d'échange et de mémoire qui procède sans cesse à des ajustements, à des reconfigurations, à des adaptations et des actualisations ${ }^{10}$. Mais les contes ne sont pas pour autant situés hors du temps. Leur émergence sur la scène littéraire occidentale correspond à une certaine situation historique - non pas la toute fin du XVII siècle (à Paris), comme on pourrait le croire, mais entre le mitan du XVI ${ }^{\mathrm{e}}$ siècle (à Venise, avec Giovan Francesco Straparola) et la première moitié du XVII ${ }^{\mathrm{e}}$ siècle (à Naples, avec Giambattista Basile). Ces histoires passent en France peu après dans les traductions de Jean Louveau et Pierre de Larivey ${ }^{11}$ qui seront lues dans les salons parisiens du Grand Siècle par Mme Murat, Mlle Lhéritier, Perrault et leurs contemporains ${ }^{12}$.

Pour différents qu'ils soient, ces contes littéraires, pris dans une dialectique entre transmission orale et écrite, tendent à être relativement ramassés et ils se différencient de formes connexes comme la novella par leur facture - à savoir une « grammaire » et des éléments sémantiques (les « motifs ») immédiatement identifiables et reconnaissables par les lecteurs/auditeurs dans la société où ils ont cours ${ }^{13}$.

${ }^{10}$ Ils se prêtent aux deux processus de rémanence identifiés par Foucault que sont (1) le transfert (transgénérique, transmédiatique...) d'un champ d'application à un autre et (2) la redistribution des énoncés dans un nouvel ensemble (Foucault, 2008 : 82-83 ; Eichel-Lojkine, $2013: 21$ ).

${ }^{11}$ Cette entreprise de traduction commence en 1560 (traduction de la première partie des Nuits par Louveau, publiée à Lyon, G. Rouillé) et connaît une étape importante en 1585 avec l'édition conjointe de la première partie (traduite par Louveau, revue par Larivey) et de la seconde partie (traduite par Larivey) (Straparola G. F., 1585). Éd. moderne : Straparola (1857). Adaptation récente : Straparola (1999). Pour plus de détails sur les éditions de ce texte, voir Eichel-Lojkine (2013: 131-149).

${ }^{12}$ Sur ce processus intertextuel délibéré, voir Heidemann \& Adam $(2010: 51)$.

${ }^{13} \mathrm{Au}$-delà de ces quelques éléments, les premiers contes écrits italiens et français ne constituent pas un ensemble homogène. Il n'y a pas de constante, d'invariant, en ce qui concerne leur articulation ou leur rupture par rapport à la tradition orale ; leurs sujets, leur teneur, leur style, leurs modes d'énonciation; leur mode de production, de communication et de réception ; leur usage social (instruction ou divertissement) ; leurs destinataires (divers pour leur condition sociale, leur sexe, leur âge) ; leur édition (en histoire séparée ou en anthologie, en livre ou en revue avec le Mercure galant) et impression (soignée ou sur papier bleu); ou encore en ce qui concerne la terminologie qui leur est appliquée jusqu'à Grimm (exempla, novelle/nouvelles, histoires, historiettes, contes de fées, märchen). 
Leur identité si spécifique, la magie qu'ils opèrent (ce que les frères Grimm nomment leur essence poétique dans leur préface de 1812), la fascination durable qu'ils exercent tiennent sans doute à leur poétique «simplexe». Le terme simplexe employé comme adjectif pour décrire des objets ou des processus ${ }^{14}$ est apparu récemment, d'abord dans le contexte des sciences du vivant, puis le spécialiste de l'évolution Alain Berthoz en a généralisé et vulgarisé l'usage en France. Un des exemples qu'il donne est la subtilité des mécanismes qui répartissent le développement des fonctions (sensorielles, motrices, cognitives) selon un calendrier assurant leur emboîtement, leur ordonnancement selon des «périodes critiques » de développement (Berthoz, 2009 : 82-85). De nombreuses capacités sensori-motrices ne se mettent pas en place chez les bébés animaux de façon continue, mais de manière échelonnée - par exemple la période critique correspond aux premières semaines pour le développement des propriétés des cellules du cortex visuel. Cette invention de l'évolution est simplexe en ce qu'elle combine la complexité des processus qui sous-tendent la période critique et la simplicité d'un calendrier échelonné, qui simplifie la relation de l'être vivant au monde extérieur en évitant à son cerveau d'avoir à établir en parallèle les différents réseaux fonctionnels.

Est simplexe, donc, non pas ce qui peut se décomposer en un nombre limité d'éléments simples, non pas ce qui s'oppose à la complexité, mais ce qui recourt à des processus d'une grande complexité pour un résultat élégant, rapide, efficace, optimal. C'est à un résultat de ce type qu'aboutit l'organisation du conte qu'on peut définir comme un étagement de trois niveaux herméneutiques (Eichel-Lojkine, 2013 : 225).

(1) Le premier niveau est le niveau structural de la construction de la narration, du schéma narratif, souvent fondé sur des couplages d'actions (interdiction/transgression, accord/refus...). C'est sur ce niveau que se sont focalisées les analyses formalistes et structurales au détriment des autres aspects de la poétique des contes $^{15}$ - avec des conséquences assez regrettables en milieu scolaire ${ }^{16}$. Avec la distance, on comprend que ces études ont fait progresser la connaissance des contes essentiellement en portant l'attention vers la double face du motif :

\footnotetext{
${ }^{14}$ Employé comme nom commun (un simplexe), le terme a par ailleurs un sens très précis en géométrie et désigne également un type d'algorithme.

${ }^{15}$ La célèbre Morphologie du conte (1928) part bien d'un corpus précis (les contes russes d'Afanassiev), mais tend à synthétiser l'ensemble des contes populaires, voire l'ensemble des contes connus en une seule formule synthétique. Ce grand proto-récit virtuel est constituée d'une concaténation rigide de séquences-types abstraites dans lesquelles se dissout le sémantisme proprement dit des motifs constitutifs. Sur les critiques que ce modèle a suscitées, voir (entre autres) Eichel-Lojkine (2013 : 412-414).

${ }^{16}$ Serge Martin (1997) et Marie-Agnès Thirard (2004) se sont élevés contre les pratiques pédagogiques sclérosantes enfermées dans le carcan du schéma quinaire (découpage narratif en cinq étapes) hérité de Propp.
} 
son contenu sémantique (par exemple, le don d'une étoile d'or) et sa fonction dans la progression du récit (l'étoile en question peut tenir lieu d'objet magique mis à la disposition du héros par un donateur surnaturel ou un auxiliaire, ou bien de bienfait récompensant le héros après une épreuve, par exemple dans Le tre fate de Basile ${ }^{17}$ ).

Au niveau de la structure, la simplicité tient aux éléments suivants :

1 - au caractère sobre d'une narration dont Max Lüthi qualifie le style d'abstrait, de formel, de diagrammatique (Lüthi, 1986 : 24-36) ; à son caractère économique, qui évite les péripéties inutiles ;

2 - à la clarté des oppositions radicales qui y sont convoquées (Lüthi, 1986 : $34)^{18}$;

3 - au personnel narratif stéréotypé (Lüthi, $1986: 72)^{19}$;

4 - à la netteté des séquences, des motifs qui constituent autant de briques isolées que le récit se charge d'articuler, de combiner, d'enchaîner, d'interconnecter (Chap. 4 : Isolation and Universal Interconnection; Lüthi, 1986 : 37-40);

5 - à l'achèvement du récit merveilleux, qui procure un sentiment de satisfaction au lecteur du fait que le poids de la fatalité qui pesait sur les épaules du héros y est levé ${ }^{20}$. Analysant le conte en tant que «forme simple», André Jolles évoque le caractère complet, révolu du parcours narratif, qui correspond à une certaine « disposition mentale »: la « morale ingénue » veut que l'oppression, la misère, la détresse initiales soient renversées, l'injustice réparée (Jolles, 1970 : 173-195 ; Sermain, 2010b : 195).

(2) Le deuxième niveau, le niveau figuratif, est constitué des réseaux souterrains d'images à valeur fantasmatique qui tissent leur toile indépendamment du fil narratif. Ils exploitent le potentiel suggestif de motifs traditionnels choisis pour leur puissance imaginaire. Cannibalisme, amputation, castration, dévoration, humiliation dégradante, déshumanisation, souffrance muette, anéantissement, boucheries, dépossession, amours déviantes et violentes sont au programme. Comme le dit François Flahault à propos des contes populaires qu'il analyse (en anthropologue versé dans la psychanalyse), «un récit parfaitement moral, c'est-à-dire sans violence, sans démesure, sans aucune expression du mal

${ }^{17}$ Les trois fées (Basile, 2002 : 292).

${ }^{18}$ Par ex. beau vs. monstrueux, aînés vs. cadet, préféré vs. rejeté, charitable vs. méchant, fiancée blanche $v s$. fiancée noire, palais $v s$. masure, roi vs. paysan comme ce «pauvre paysan, vraiment très pauvre, si pauvre qu'il n'avait aucun espoir, malgré toute la peine qu'il endurait, de se dorer une miche » sur lequel s'ouvre Lo turzo d'oro (L'arbre d'or) de Basile (2002 : 421).

${ }^{19}$ Il faut concevoir les personnages des contes comme des figures pourvues d'un rôle, plus que comme des types stylisant des modèles réels.

${ }^{20}$ Mais la virtualité tragique de l'histoire est au contraire confirmée dans les contes d'avertissement. 
retirerait du même coup à ses auditeurs, lecteurs ou spectateurs la racine du plaisir qu'ils en tirent » (Flahault, 2001: 45).

Les connotations agressives ou érotiques de certains motifs qui se font écho génèrent une large gamme de sentiments chez le lecteur - fascination, plaisir par procuration, angoisse, terreur. Ces images se structurent en réseaux à partir de deux principes : l'opposition, la différenciation, d'une part et, symétriquement, la correspondance, la ressemblance ${ }^{21}$.

3) Le conte n'est pas seulement un schéma narratif ou un creuset de fantasmes. Il est en prise avec la vie sociale - aussi bien avec des rites, des coutumes, des pratiques magiques qu'avec les mœurs et le système social (la vie de cour, les mariages forcés, les remariages, le pouvoir absolu, la pauvreté, le veuvage, l'héritage, l'abandon d'enfants, les conflits fraternels, la loyauté entre maître et valet, la fidélité entre frère et sœur etc.) (Chap. 5 : Sublimation and All-Inclusiveness ; Lüthi, 1986 : 66-69) ${ }^{22}$.

Le troisième niveau du conte concerne cette articulation au « réel » que Lüthi décrit comme un processus de sublimation de la matière. Il s'agit du niveau symbolique de la «pensée », de la philosophie du conte. Le sens d'un conte ne se résout pas dans une morale (énoncée à l'ouverture ou à la fin de l'histoire), dans une idéologie explicite. Les contes invitent plutôt à penser une contradiction symbolique entre deux codes ou deux systèmes de croyance. Comme dans le mythe, cette contradiction ne peut pas se dire directement, sans employer la voie d'un récit qui se déplie. Dans Le Maître Chat, semblent s'affronter deux modèles, un modèle conformiste et un paradigme "révolutionnaire ${ }^{23}$. L'ordre $\mathrm{y}$ est à la fois posé et aboli. On y interroge, plus que dans les textes parallèles de

${ }^{21}$ Ainsi Le Maître Chat de Perrault est-il parcouru par des couplages d'opposés qui fonctionnent par binômes : [vêtu, paré vs. dénudé, déshabillé, écorché] ; [nourri vs. anéanti] ; [vide $v s$. plein] ; [endroit $v s$. envers], ou par groupe ternaire : [allongé $v s$. dressé $v s$. pendu].

Parallèlement, se tisse un réseau de figures équivalentes: [bottes//manchon]; [dépouillé de son argent//de ses habits] etc. Pour une analyse développée des principaux motifs du Maître Chat sous cet angle, voir Eichel-Lojkine (2013: 238-275).

${ }^{22}$ Marie-Agnès Thirard (2004: 152-153) rend compte d'un travail sur le niveau du symbolique dans le conte lorsqu'elle évoque les réactions d'élèves migrants aux thèmes du mariage forcé et de l'exercice du pouvoir absolu dans La Belle aux Cheveux d'Or de Mme d'Aulnoy et des textes africains parallèles.

${ }^{23}$ Selon un schème conformiste, l'histoire assigne à chaque ordre son rôle (maître/serviteur ; puissants/misérables) et distribue à chaque frère un bien selon son rang de naissance (le moulin, l'âne et le chat). Mais ce partage se révèle bien moins équilibré, convenable, que celui auquel on voit régulièrement les héros de contes procéder eux-mêmes, lorsqu'ils ont à départager trois prédateurs qui se disputent les restes d'une proie dans une forêt (par exemple dans Fortunio de Straparola, III, 4). L'histoire peut donc symétriquement apparaître comme une illustration du désordre. Elle retrace le parcours perturbé, chaotique, plein de méandres et de détours, d'un aventurier qui se fraie un chemin au moyen des ruses, des mensonges, des chantages de son animal serviable, sans reculer devant la mésalliance et le renversement des hiérarchies instituées. 
Straparola et de Basile ${ }^{24}$, le décalage entre les différentes hiérarchies. La hiérarchie entre les règnes (humain/animal), entre les positions (maître/serviteur), entre les conditions (roi/vilain sans ressource) ne correspond pas à une différence d'intelligence («Carabas» est niais), de perspicacité (le roi se fait berner par un chat), d' « industrie » et de « savoir-faire», et c'est ce qui jette le trouble chez le lecteur.

Cette philosophie, lorsqu'elle est perceptible, se dit dans le conte de manière bien plus subreptice que dans la fable. Ainsi, le sophisme qui ouvre Le Loup et l'agneau de La Fontaine ( $«$ La raison du plus fort est toujours la meilleure $\left.{ }^{25} »\right)$ invite explicitement à une réflexion morale et politique sur la possibilité de vivre en société, de manière réglée, avec des hommes-loups prêts à tout pour servir leur propre intérêt, pour satisfaire leur appétit individuel (Sermain, 2010b : 194). $\mathrm{Ni}$ les proverbes traditionnels que cite parfois Straparola ou qui servent de clausules aux contes de Basile, ni les moralités des contes de Perrault souvent décalées par rapport à l'histoire ne répondent à une telle stratégie.

\section{DYSFONCTIONNEMENT DE LA «SIMPLEXITE »}

La magie du conte n'opère que si le geste littéraire respecte, assume cette triple dimension, cet enclenchement entre trois niveaux. Les cas contraires sont fréquents dans l'histoire littéraire et passionnants à explorer. Voici deux des aspects que peut prendre une intervention littéraire qui fait perdre au conte sa simplexité :

1 - la désarticulation de l'engrenage [structural-figuratif-symbolique]: ce processus est à l'œuvre dans les homélies médiévales qui réemploient les structures narratives folkloriques pour réveiller les paroissiens «quand fatigués et frappés d'ennui ils commencent à sommeiller », selon les propos du prédicateur du XIII ${ }^{\mathrm{e}}$ siècle Jacques de Vitry ${ }^{26}$. Des contes populaires, des histoires de pactes avec le diable, de cigognes adultères, d'une main qui change de couleur (autant d'exemples tirés de sermons d'Étienne de Bourbon) (Schmitt, 1985 : 83-92) sont utilisés de manière pragmatique soit comme des aiguillons pour réveiller et attiser l'attention des laïcs, soit comme supports à une leçon édifiante, à une période

${ }^{24}$ Respectivement Costantino Fortunato, XI, 1 et Cagliuso, II, 4.

${ }^{25}$ Fables, I, 10, v. 1.

${ }^{26}$ «Sans la science des Écritures, en effet, nous ne devons pas avancer d'un pied. Cependant, pour réveiller et aiguiser l'attention des laïcs, il faut y insérer des exemples accessibles à tous, mais qui toutefois leur apportent quelque édification, afin d'éviter que cette parole prophétique ne nous soit objectée: Des gens iniques m'ont raconté des histoires [fabulationes] et non ta loi [Narraverunt mihi iniqui fabulationes, sed non ut lex tua (Ps. 118 : 85)] ». (Traduction française du texte latin de Vitry par M.-C. Gasnault ; Schmitt (dir.), 1985 : 51-52). 
où l'on fait confiance aux récits pour changer les comportements ${ }^{27}$. Ce qui intéresse les prédicateurs dans les contes, ce n'est pas leur simplexité, mais leur brièveté, leur caractère familier, divertissant, simple, accessible à tous, qui fait d'eux des « alliés objectifs » du sermon.

2 - la complexification de la structure narrative : ce processus, propre aux contes galants, peut générer, par réaction, un désir de simplification, de "naïveté», d'authenticité (comme chez Perrault) qui peut aller jusqu'à l'appauvrissement de la matière. Ce sont ces jeux entre galanterie et "naïveté » dans les contes de fées français de la fin du XVII ${ }^{\mathrm{e}}$ siècle que nous nous proposons d'explorer plus avant.

\section{LES JEUX DE LA GALANTERIE ET DE LA «NAÏVETE »}

\section{Simple et naturel}

Chez les Classiques, le simple désigne ce qui est ou parait naturel, non affecté, non apprêté, en l'espèce le discours qui rejette le pédantisme ou l'esthétique mondaine. C'est ainsi que la rhétorique ancienne pouvait rejeter, au nom du plus sobre atticisme, l'asianisme exubérant, ampoulé, maniéré, virtuose qui faisait des orgies de pointes, d'antithèses, d'accumulations, de jeux d'esprit (Curtius, 1986, t. I : 129).

Le discours simple tend à effacer les traces de littérarité en son sein et cherche éventuellement à atteindre au sublime par ce moyen. C'est dans ce contexte que le stéréotype littéraire (hérité d'Apulée et de Boccace) de la vieille racontant des fables à une enfant apeurée ${ }^{28}$ fait retour - avec les mies et les nourrices à la place de la vieille au service des brigands, et les «moindres familles » aussi bien que les enfants dépourvus de raison, à la place de la jeune Carithée. Le dispositif se raffine lorsque l'Académicien propose une nouvelle variante, inédite, qui implique son fils. Pour faire la promotion du simple en littérature et défendre ses positions de Moderne, il imagine qu'un jeune auteur prend la place des parents et des mies pour s'adresser à un public simple : «On ne trouvera pas étrange qu'un Enfant ait pris plaisir à composer les Contes de ce recueil [...]» (Préface de 1697, « À Mademoiselle »; Perrault, $1991: 89$ ).

${ }^{27}$ Ce n'est donc pas sous son aspect proprement littéraire que le Moyen Âge a d'abord connu le conte. Voir Velay-Vallantin (1992: 28) : « [...] la matière du conte se rencontre dans d'autres textes médiévaux, sous forme de structures narratives folkloriques : le conte est utilisé à des fins didactiques, comme la prédication religieuse ou l'éducation des princes ». Voir aussi Bremond (1998: 21-42).

${ }^{28}$ Au livre IV des Métamorphoses ou L'Âne d'or d'Apulée et au livre XIV de la Généalogie des dieux de Boccace. 
Cette «scénographie pseudo-naïve » a été démontée par Ute Heidmann notamment (Heidmann, 2011: 46). Perrault rêve d'une poétique proche de l'enfance, fuyant en tout cas le pédantisme pesant des régents de collège déjà moqué par Montaigne ( Du pédantisme », I, 25). Une telle poétique rivaliserait de saveur, de fraîcheur, de "naïveté » avec la tradition. Sa première préface (1695) se construit sur une opposition entre les hommes qui se veulent graves et qui n'ont que mépris pour des récits dépourvus à leurs yeux de substance, d'apprêt, d'élégance, d'une part, et les gens de bon goût, les gens vraiment distingués qui n'ont rien en commun avec les demi-savants, d'autre part ${ }^{29}$.

Dans le même ordre d'idées, l'Abbé de Villiers, dans ses Entretiens sur les contes de fées (1699), associe le conte à l'art suprême de la simplicité qui est censé être le fait des nourrices. Ces Entretiens se composent d'un dialogue entre le Parisien et le Provincial qui entrent parfois en conflit, mais s'accordent en tout cas à vanter une telle poétique : "car la simplicité et le naturel de la narration, est ce qui fait le principal mérite d'un conte ${ }^{30} \gg$. Imiter la simplicité des nourrices, ce n'est certainement pas parler comme elles, mais plutôt les citer avec des guillemets, avec des pincettes, avec des précautions qui atténuent la crudité de leur langage et la bêtise d'histoires plus ou moins bien fagotées. Le style " naïf » se construit donc à partir de deux repoussoirs : les contes de bonnes femmes et les contes trop mondains des fées modernes.

\section{Le repoussoir du conte galant}

Les premiers contes de fées français - à commencer par Peau d'Âne, le tout premier conte merveilleux de Perrault et le seul publié sous son nom (1694) sont indéniablement et indissociablement liés au monde de la galanterie et à la sphère mondaine (Viala, 2008; Viala 2009) ${ }^{31}$. Nous prendrons ici comme

${ }^{29}$ Préface de 1695 : «La manière dont le Public a reçu les Pièces de ce Recueil, à mesure qu'elles lui ont été données séparément, est une espèce d'assurance qu'elles ne lui déplairont pas en paraissant toutes ensemble. Il est vrai que quelques personnes qui affectent de paraître graves, et qui ont assez d'esprit pour voir que ce sont des Contes faits à plaisir, et que la matière n'en est pas importante, les ont regardées avec mépris ; mais on a eu la satisfaction de voir que les gens de bon goût n'en ont pas jugé de la sorte » (Perrault, $1991: 3$ ).

${ }^{30}$ À ce propos du Parisien, le Provincial renchérit en citant comme modèles les contes de Perrault : «Cependant vous m'avouerez que les meilleurs contes que nous ayons, sont ceux qui imitent le plus le style et la simplicité des nourrices, et c'est pour cette seule raison que je vous ai vu assez content de ceux que l'on attribue au fils d'un célèbre académicien [Pierre Perrault Darmancour] » (Abbé de Villiers, Entretiens sur les contes de fées, 1699, éd. Boch). Voir L'âge d'or du conte de fées (2007: 398-400).

${ }^{31}$ Parmi les «contes galants » parus dans les années 1695-1698, on peut ranger Le Prince Rosier et Riquet à la houppe de Catherine Bernard (des contes récités par deux jeunes filles rivales dans la nouvelle Inès de Cordoue, 1696) ou Les Enchantements de l'éloquence de Mlle Lhéritier, 
exemple de conte galant Serpentin vert de Mme d'Aulnoy (Les Contes des fées, t. IV, 1697). Il est clair que la sobriété n'est pas ce qui est recherché dans ce conte, qui emprunte aux genres très élaborés de la tragédie lyrique et de la comédie-ballet $^{32}$. Le récit, qui adopte la trame usuelle des amants qui affrontent des épreuves avant que l'amour n'obtienne sa récompense, veut rivaliser avant tout avec les Amours de Psiché et de Cupidon de La Fontaine (Paris, Barbin, 1669), avec un recours massif à la Fable, à la mythologie dans ce texte ${ }^{33}$.

La matière est prise à Apulée, directement cité par un des personnages, la reine Bellotte s'adressant à sa sœur Laideronette ${ }^{34}$. La composition de La Fontaine constitue l'arrière-plan littéraire du récit, qui a aussi des points de contact avec L'arbre d'or (Lo turzo d'oro) de Basile ${ }^{35}$. Comme le note Marie-Agnès Thirard, Mme d'Aulnoy recourt aux techniques du roman baroque qui complexifient le conte (Thirard, 2004 : 151-152) ${ }^{36}$. La complication vient ici de ce que la conteuse crée un système en trompe l'œil pour faire croire que Serpentin vert est une récriture inversée de l'histoire source. Car le lecteur constate, dans un premier temps, que la prévision de Bellotte qui voit en sa sœur une autre Psyché est prise en défaut. Laideronette, qui « appréhende que son mari ne soit un monstre » ${ }^{37}$, n'a pas l'heureuse surprise de découvrir un Amour endormi, «blond, blanc, jeune et tout aimable » (Aulnoy, 2004 : 650). Mme d'Aulnoy fait un double pied de nez à La Fontaine. Elle contrevient à la tradition qui veut que l'héroïne découvre à la lumière de la lampe un mari endormi qui n'a rien d'un monstre : or Laideronnette pousse un «cri épouvantable [...] lorsqu'[...] elle vit l'affreux Serpentin Vert aux

dont le type galant éclate par comparaison avec Les Fées de Perrault, bâti sur la même trame et paru la même année (1695).

${ }^{32}$ Le couple héroïque dialogue en vers chantés ( $«$ Est-ce vous, Serpentin, cher Amant, est-ce vous ? ») et, véritable deus ex machina, le dieu Amour apparaît à la fin dans une scénographie spectaculaire (Serpentin vert ; Aulnoy, 2004 : 638, 640, 655, 666).

${ }^{33}$ Contrairement à une idée reçue, les «Modernes » ne bannissent pas tout geste littéraire fondé sur des emprunts à la littérature classique. Ils encouragent plutôt à privilégier, parmi le patrimoine antique, les auteurs de fiction à l'imaginaire nourri par les mythes et les histoires de dieux (Apulée, Lucien) plutôt que les satiristes tranchants (Juvénal, Horace, admirés par Boileau) - cf. Viala (2009: 86).

${ }^{34}$ «Quelle erreur, s'écria la reine Bellotte, l'on dit à Psyché qu'elle avait un monstre pour époux, et elle trouva que c'était Amour [...]» (Serpentin vert; Aulnoy, 2004 : 650).

${ }^{35}$ La trop curieuse Parmettella est punie et soumise à des tâches impossibles pour avoir voulu voir le corps de celui qui la visite de nuit ; elle découvre non un corps d'esclave noir, mais un beau jeune homme : «[...] et elle, alors, prenant un petit briquet qu'elle avait préparé, enflamma l'amadou, mit le feu au soufre et alluma la chandelle ; puis elle souleva la couverture et vit l'ébène transformé en ivoire, le caviar en lait crémeux et le charbon en chaux vive. Elle était là, bouche bée, à regarder ces beautés [...] » (L'arbre d'or ; Basile, 2002 : 422).

${ }^{36}$ À propos de La Belle aux Cheveux d'Or.

${ }^{37}$ Pour reprendre les mots de La Fontaine à propos de Psyché (Préface ; La Fontaine, 1990 : 39). 
longs crins hérissés » (Aulnoy, 2004 : 650). La conteuse contrevient encore aux consignes de La Fontaine qui voulait, pour des raisons de bienséance, que les lecteurs fussent informés de la véritable qualité du mari, quand bien même Psyché ne l'était pas ${ }^{38}$. Enfin, dans un jeu de miroirs, elle fait de la prévision de Bellotte qui prévoit une bonne surprise à sa sœur un équivalent inversé de l'oracle qui prévoit un époux monstrueux à Psyché ${ }^{39}$.

Naturellement, à la fin de l'aventure, on découvre que cette reprise inversée de l'histoire n'était qu'un leurre. Serpentin vert est bel et bien un «aimable époux » à « la figure charmante $»^{40}$. Serpentin est donc finalement semblable et au Cupidon de la fabella antique et au héros de L'arbre d'or - le parfait époux de Parmetella condamné par un sort à vivre dans un corps d'esclave maure.

Nous atteignons dans Serpentin vert au comble du raffinement et de la sophistication, bien loin de l'esthétique des devanciers italiens. Du coup, le conte galant prend le risque de faire écran aux puissants symboles qu'il emploie ${ }^{41}$ et de les noyer dans des descriptions de merveilles surchargées, sans autre fonction que décoratives $^{42}$. En l'occurrence, Mme d'Aulnoy prend le risque d'imiter une composition déjà complexe. Non seulement l'œuvre de La Fontaine mêle du " langage rimé » à la prose et s'accompagne de "quelques autres enrichissements » (par exemple de riches descriptions et des conversations) (Préface ; La Fontaine, 1990 : 39-40, 53) ; mais surtout elle met en place un double niveau narratif, l'histoire étant racontée par Poliphile qui lit sa nouvelle composition à trois amis à Versailles. C'est le dispositif idéal pour un effet de mise en abyme, avec des parallèles entre Poliphile qui s'interroge sur son invention tirée d'Apulée, et

38 «[...] il ne faut pas que l'on croie un seul moment qu'une si aimable personne ait été livrée à la passion d'un monstre [...] ; ce serait un trop grand sujet d'indignation au lecteur » (Préface ; La Fontaine, 1990 : 39-40).

${ }^{39}$ Le passage sur l'oracle se trouve dans Les Amours de Psiché et de Cupidon (La Fontaine, 1990 : 55) et il est commenté par La Fontaine dans sa Préface (1990 : 39).

40 « Descendant aux Enfers sous la conduite d'Amour, l'héroïne « craignait d'y rencontrer un mari sous la figure d'un serpent : mais l'Amour qui se mêle encore de rendre quelquefois de bons offices aux malheureux [...] avait ordonné que Serpentin Vert deviendrait ce qu'il était avant sa pénitence. [...] De sorte que la première chose que la reine trouva, ce fut son aimable époux; elle ne l'avait jamais vu sous une figure si charmante [...] ( Serpentin vert; Aulnoy, 2004 : 667).

${ }^{41}$ Ce n'était pas le cas chez les devanciers italiens. Straparola conservait sans l'atténuer la symbolique érotique du motif folklorique du fiancé animal (ou plutôt bestial) dans Il Re Porco (II, 1) ; Basile suggérait un accouplement monstrueux lorsqu'il décrivait la première nuit de l'héroïne avec un maure qui n'est autre qu'une variante de bête monstrueuse, dans Lo turzo d'oro $(\mathrm{V}, 4)$. Voir à ce sujet Lüthi (1986 : 70).

${ }^{42}$ De son balcon, Laideronette « découvrit toutes les beautés que la nature, secondée par l'art, peut ménager sur la terre : des jardins remplis de fleurs, de fontaines, de statues et d'arbres rares, des forêts en éloignement, des palais dont les murs étaient ornés de pierreries, les toits de perles [...]» (Serpentin vert; Aulnoy, 2004 : 638-640). 
l'auteur de la Préface qui raisonne «sur le genre d'écrire qu' [il a] choisi » (La Fontaine, $1990: 38)^{43}$.

Aussi le conte prend-il le risque de s'empêtrer dans les méandres d'une narration à rallonge et dans les complications d'une superposition d'intertextes tout à la fois exhibés, récusés et pris pour modèles. Perrault n'était pas insensible au charme de Psyché et Cupidon, à ses récritures, imitations et prolongements. Outre sa première préface aux Contes, son Parallèle en témoigne (Perrault, 1692: 293 $)^{44}$. Cependant, il est conscient des excès auxquels la dialectique mondaine entre l'imitation et la différenciation peut mener. C'est peut-être en réaction à ces complications artificielles que, dans un conte comme Les Fées, il simplifie son récit à l'extrême, quitte à l'appauvrir.

\section{LE RISQUE DE L'APPAUVRISSEMENT}

Les Fées est un conte à la structure simple qui, pour cette raison, fait partie du répertoire que les éditeurs modernes destinent aux enfants (fig. 2a, b).

Cette histoire n'a pas d'équivalent dans la collection de Straparola, mais peut être rapproché de deux contes de Basile. Le tre fate (Les trois fées) part de l'opposition entre deux demi-sœurs, la gracieuse Cicella et la repoussante Grannizia adorée de sa mère ${ }^{45}$. Le die pizzele (Les deux petites pizzas) oppose deux cousines, la courtoise Marziella et la désagréable Puccia, elle aussi chérie d'une mère dont elle est tout le portrait ${ }^{46}$.

Ces histoires appartenaient au répertoire traditionnel des contes d'avertissement. Perrault s'en empare et produit une narration très schématique fondée sur

${ }^{43}$ Voir à ce sujet, Heidmann $(2001: 49)$.

${ }^{44}$ Voir Eichel-Lojkine (2013: 32).

${ }^{45}$ Le tre fate (III, 7) développe l'argument du « châtiment d'une femme envieuse, qui, voulant faire trébucher sa belle-fille, ne réussit qu'à la porter au firmament ». Récompensée par les fées pour sa courtoisie, Cicella, de retour auprès de sa belle-mère, est envoyée "garder les cochons vêtue d'un simple sarrau à même sa peau nue ». Elle rencontre là un grand seigneur qui demande sa main ; la marâtre veut lui substituer sa propre fille et enferme Cicella dans un tonneau pour l'ébouillanter. Le fiancé découvre la traîtrise, échange les deux filles, s'en va sur ses terres avec Cicella, de sorte que la marâtre, de retour avec un gros fagot, ébouillante et découenne sa fille Grannizia avant de se jeter elle-même dans un puits.

${ }^{46}$ Dans Le die pizzele (IV, 7), «Marziella se montre courtoise avec la petite vieille» (en lui donnant sa petite pizza), vieille «qui, en récompense, lui accorde un don » : "Que chacun de tes souffles laisse échapper de ta bouche des roses et des jasmins, que de tes cheveux, à chaque coup de peigne, tombent perles et grenats ». Une conduite inverse de la part de Puccia lui rapporte une chevelure infestée de bêtes. Il est question de marier Marziella avec le fils du roi, mais sa tante, « jalouse de sa bonne fortune », veut lui substituer sa propre fille et elle prépare traitreusement un méfait dont l'héroïne (jetée à l'eau et retenue captive par une sirène) sort finalement vainqueur. 
a

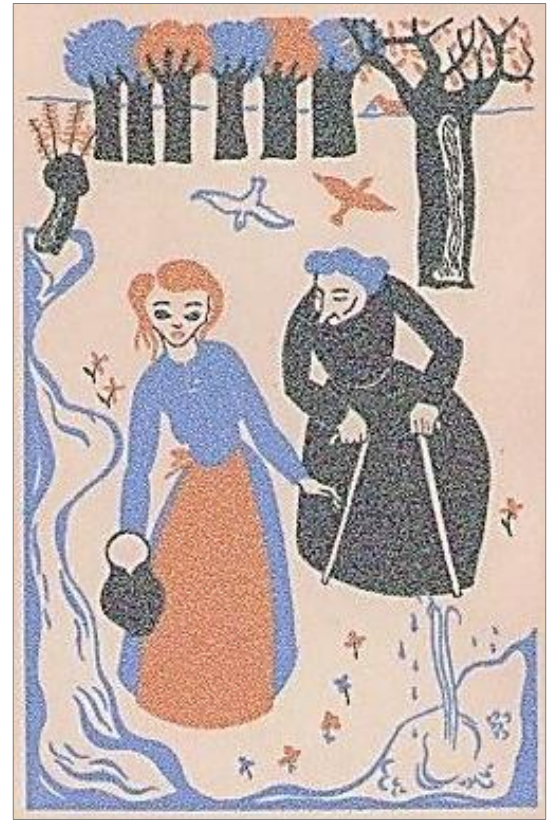

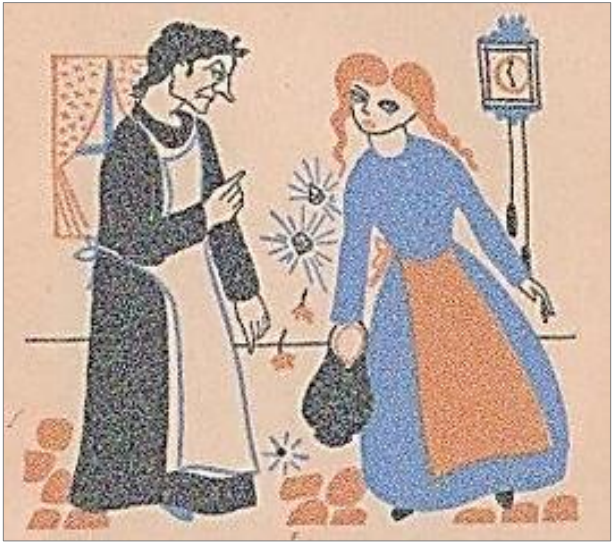

Fig. 2a, b. Charles Perrault et A. Linglet (ill.), Contes de ma Mère l'Oye (avec trois contes de Mme d'Aulnoy), éditions de la Toison d'or, sl, sd (années 1950), In-12 : La cadette puise de l'eau pour la vieille appuyée sur deux cannes / La mère gronde la cadette de revenir si tard au logis et la fille lui répond en déversant à chaque parole fleurs, diamants et perles

un strict parallélisme entre les deux sœurs rivales ${ }^{47}$. Ce conte, fort répandu, a des parallèles africains ${ }^{48}$ et on y reconnaît la «structure en miroir » décrite par l'ethnologue Denise Paulme :

Il arrive encore qu'à la progression ascendante due à la conduite satisfaisante du héros dans une première partie succède une autre partie symétrique descendante, où un deuxième actant (l'anti-héros), voulant imiter le premier mais ne cherchant que sa satisfaction personnelle, adopte une conduite opposée, ignore les conseils ou les ordres reçus et se trouve à la fin puni en conséquence : la présence de deux parties symétriques donne au récit une structure en miroir (Paulme, $1976: 25)^{49}$.

47 «Ainsi dans un conte assez négligé de Perrault, Les Fées, chacune des deux sœurs agit selon les qualités qui lui sont échues dans la famille, et celles-ci ne valent que dans une opposition qui structure leur histoire et conduit l'une à la cour et au trône, et l'autre à une mort de brute abandonnée dans la forêt » (Sermain, 2010b : 195).

${ }^{48}$ Marie-Agnès Thirard (2004) cite notamment une version tunisienne, Hafsa, à chaque mot, un pet (Ben Hassen \& Charny 1997 : 81-90).

${ }^{49}$ La description est approfondie ensuite (Paulme, 1976 : 38-43). 
Dans Les Fées, le double portrait contrasté des deux sœurs (l'aînée ressemble à la mère acariâtre, la cadette au doux père décédé) est complété par la mention d'un traitement inégalitaire de la part de la mère - traitement qui a sa logique d'un point de vue psychologique (la mère aime qui lui ressemble ${ }^{50}$ ), mais qui ressortit du chiasme et de l'oxymore pour le lecteur (l'amour se porte sur la désagréable, l'aversion sur l'aimable). Le test de la vieille à la fontaine valide le statut d'héroïne de la cadette ${ }^{51}$ et donne le coup d'envoi à une trajectoire ascendante pour la cadette méprisé ${ }^{52}$, à une trajectoire descendante pour l'aînée choyée ${ }^{53}$.

Comme pour se rapprocher d'un modèle simple, sobre, authentique, Perrault a ici mis en sourdine l'art du badinage cher à La Fontaine ${ }^{54}$, ses notations malicieuses, sa façon de faire percer la voix du conteur mondain sous la voix ancestrale de la tradition ${ }^{55}$.

La critique a surtout retenu la simplicité du canevas narratif, s'est livrée à des jugements sévères à son propos et l'a confronté à "Dame Holle » de Grimm: "Une veuve avait deux filles, l'une jolie et courageuse, l'autre paresseuse et laide... ». Pour Soriano et Simonsen, Perrault transforme en parabole

${ }^{50}$ «Comme on aime naturellement son semblable, cette mère était folle de sa fille aînée, et en même temps avait une aversion effroyable pour la cadette » (Perrault, 1991 : 147).

${ }^{51}$ «Un jour qu'elle était à cette fontaine, il vint à elle une pauvre femme qui la pria de lui donner à boire » (Perrault, $1990: 148)$.

${ }^{52}$ La cadette est expulsée du foyer comme responsable de la malédiction qui afflige sa sœur aînée. Cette expulsion se révèle bénéfique pour elle puisqu'elle rencontre dans les bois le fils d'un roi qui «en devint amoureux », «l'emmena au Palais du Roi son père, où il l'épousa » (Perrault, $1990: 149)$.

${ }^{53}$ Comme la cadette est retournée au foyer avec le don de faire sortir de sa bouche une fleur ou un pierre précieuse à chaque parole, l'aînée est immédiatement dépêchée à la source par sa mère. Elle y subit un contre-test de la part de la fée qui a changé d'apparence et ne se présente plus comme une vieille, mais comme une dame magnifiquement parée : "Est-ce que je suis venue ici, lui répondit cette brutale orgueilleuse, pour vous donner à boire [...] ?» (Perrault, $1990: 148$ ). L'aînée se met à cracher des serpents et des crapauds, elle est finalement chassée du foyer, comme la cadette, mais son sort est beaucoup moins enviable : « et la malheureuse, après avoir bien couru sans trouver personne qui voulût la recevoir, alla mourir au coin d'un bois » (Perrault, 1990 : 149).

${ }^{54}$ Par exemple, Psyché, enlevée par Zéphyre, est vêtue d'une robe couverte de diamants et de pierreries - et le narrateur de préciser malicieusement : «il est vrai que c'était ouvrage de fée, lequel d'ordinaire ne coûte rien » (La Fontaine, 1990:60). Cet art du badinage est en outre théorisé par La Fontaine dans sa Préface.

${ }^{55}$ L'humour, qui est sa marque de fabrique, n'est toutefois pas totalement absent de ce texte de Perrault. Ainsi, la cadette est censée jeter fleur et pierre précieuse en parlant, mais bientôt il n'est plus question que de perles et de diamants - et les fleurs sont oubliées. Le prince qui recueille la réprouvée l'épouse non sans arrière-pensée, « considérant qu'un tel don valait mieux que tout ce qu'on pouvait donner en mariage à un<e> autre » (s.v. Fées (Les) : «il faudrait dire une autre ») (Perrault, 1989). 
moralisatrice un conte d'avertissement traditionnel qui, exploité par Grimm, se révélera très riche d'un point de vue imaginaire et fantasmatique ${ }^{56}$.

Chez Perrault, la narration est tellement épurée qu'on n'y retrouve ni la verve de Basile ${ }^{57}$, ni la présence de motifs caractéristiques du type AT. 480 (The Spinning-Women by the Springs ou The Tale of the Kind and Unkind Girls) ${ }^{58}$ :

- l'assignation de tâches impossibles aux deux sœurs,

- l'aide d'animaux serviables,

- la chevelure des fées à démêler ${ }^{59}$,

- le choix entre trois coffrets ${ }^{60} \ldots$

Concourent aussi à l'impression de pauvreté l'effacement de la tension entre liens de sang et liens d'alliance qui était présente dans la version de 1695 avec la figure de la marâtre et qui disparaît dans le texte de 1697 où il est question d'une veuve avec deux filles biologiques, semble-t-il. Mais cette disparition laisse des traces, ce qui explique que le début du texte ne soit pas clair ${ }^{61}$. Il arrive donc qu'un conte «simplexe» retourne à l'état de «simple». Dans Les Fées, cela passe par le renforcement de la structure en miroir du récit, qui est la plus élémentaire qui soit ${ }^{62}$.

${ }^{56}$ Voir Róheim (1992 : 181-192). Pour Michèle Simonsen (1992: 82), il s'agit d' « un conte tout pétri d'images et de symboles renvoyant à la toute petite enfance ».

${ }^{57}$ Le «même » conte, chez Basile, non filtré par la décence classique, présentait l'antithèse entre les deux demi-sœurs avec une verve et une emphase drolatiques qui peuvent faire penser aux blasons du beau et du laid tétin, ou à d'autres jeux sur les codes et leurs détournements parodiques et satiriques fort prisés à la Renaissance. Grannizia, le laideron au crâne pouilleux, à la tignasse ébouriffée, aux tempes déplumées, qui fait les délices de sa mère la veuve Caradonia, est un concentré de laideurs, alors que Cicella, la fille de Micco Antuono, semble sortie de la palette d'un peintre (Les trois fées; Basile, $2002: 289$ ).

${ }^{58}$ C'est pourquoi, «la simplicité à la limite la pauvreté de style des Fées ne saurait en aucun cas être mise sur le compte de sa fidélité à une éventuelle source orale » pour Michèle Simonsen (1992 : 80-87), répondant à Soriano qui voit dans « la tendance à la réitération » une «tendance naturelle à l'art oral » (s.v. Fées (Les), Perraullt 1989). Le type en question, The Kind and Unkind Girls, comprend lui-même deux grands sous-types $(1$ - Following the river sub-type auquel on rattache The drink of water group / 2 - The Encounters en route sub-type). Voir Roberts (1994).

${ }^{59}$ Un motif présent chez Basile (Les trois fées; Basile, 2002 : 291-292).

${ }^{60}$ Un motif équivalent est présent dans Les trois fées (III, 7) (Basile, 2002 : 291-293) : entre des vêtements magnifiques, l'hérö̈ne choisit une guenille; entre plusieurs portes, elle choisit celle de l'écurie pour sortir, mais les fées la font passer par une porte d'or massif - alors que la mauvaise fille fait toujours le choix inverse et reçoit sur sa tête un testicule d'âne. Plus généralement, ces quatre motifs sont également absents de la version de Grimm.

${ }^{61}$ Certes, on nous présente « une veuve qui avait deux filles », mais celle-ci forme couple avec son aînée et physiquement et moralement, comme le confirme l'emploi du pluriel («Elles étaient toutes deux si désagréables...»). De la cadette, on dit qu'elle « était le vrai portrait de son Père » (mais ne devrait-on pas dire de leur père, si c'est bien le même pour les deux sœurs ?).

${ }^{62}$ Cette structure est même plus élémentaire que le modèle du sablier qui croise les itinéraires de deux rivaux qui ont deux démarches opposées. Un tel modèle est illustré par l'une des sources 


\section{CONCLUSION}

Dans un article fondateur sur la nouvelle italienne, H. H. Wetzel émettait l'hypothèse qu'un des trois types de nouvelles, la nouvelle-aventure, avait donné naissance au conte féerique par le biais d'une de ses variantes, l'aventure merveilleuse $^{63}$. La nouvelle-aventure met au cœur du récit non pas la providence qui régissait l'ordre chrétien et féodal, mais le hasard (Fortuna) avec ses répercussions sur la vie humaine. Sa variante merveilleuse souligne, avec le rôle central dévolu à l'aide magique, le renoncement à toute prise sur la réalité, ce qui correspondrait à une époque de grande instabilité (Wetzel, 1981: 75). Le renversement de la situation initiale défavorable au héros se fait, non pas grâce à ses propres talents (comme dans la nouvelle-beffa) ou grâce à ses mérites ou à l'exercice de la vertu (comme dans la nouvelle-exemple), mais grâce à une intervention magique extérieure ${ }^{64}$.

La simplicité (ou plutôt simplexité) qu'on peut reconnaître à la forme, au « geste verbal ${ }^{65}$ » du conte merveilleux littéraire dérive donc d'un processus long et complexe. Son émergence à partir d'une ramification d'une forme savante, la nouvelle, n'a pas fini de nous émerveiller, plus de quatre siècles plus tard.

de Perrault pour son Maître Chat, Costantino Fortunato de Straparola (XI, 1). L'ascension de Costantino (qui n'héritait pourtant que d'une chatte à la mort de sa mère, la pauvre veuve Soriane) croise la chute de ses deux frères (qui avaient, eux, hérité d'une huche à pétrir le pain et d'un tour sur lequel on tourne la pâte et avaient refusé de partager leurs ressources avec leur cadet). À la fin, Costantino et ses frères se trouvent donc avoir échangé leurs positions.

${ }^{63}$ Les deux autres types sont la nouvelle-exemple et la nouvelle-beffa (Wetzel, 1981 : 41-80). Pour un exposé plus approfondi des conclusions de Wetzel, voir Eichel-Lojkine (2013 : 127-130).

${ }^{64}$ «Le succès n'est pas le fruit de ses mérites ni de ses facultés individuelles comme dans la nouvelle, mais d'une décision arbitraire, incontrôlable et hétéronome » (Wetzel, 1981 : 73).

${ }^{65}$ L'expression est d'André Jolles (1970). 


\section{BIBLIOGRAPHIE}

L'âge d'or du conte de fées : De la comédie à la critique (1690-1709). La Fée Bienfaisante et autres comedies (2007). Éd. Nathalie Rizzoni. Entretiens sur les contes de fées et autres textes critiques. Éd. Julie Boch. Paris : Champion.

Aulnoy, Marie-Catherine (2004). Contes des fées. Éd. Nadine Jasmin. Paris : Champion.

Basile, Giambattista (2002). Le Conte des contes ou Le divertissement des petits enfants. Trad. et éd. Françoise Decroisette. Strasbourg : Circé.

Ben Hassen, Bochra \& Charnay, Thierry (1997). Contes merveilleux de Tunisie. Paris : Maisonneuve et Larose.

Berlioz, Jacques \& Polo de Beaulieu, Marie-Anne (2008). L'irruption de la matière orientale dans la littérature exemplaire, $\mathrm{XIII}^{\mathrm{e}}-\mathrm{XIV}^{\mathrm{e}}$ siècle : de la description à la quantification. In Frédéric Bauden, Aboubakr Chraïbi, Antonella Ghersetti (éd.), Le répertoire narratif arabe médiéval, transmission et ouverture [Texte imprimé] : actes de Colloque international (Liège, 15-17 septembre 2005) (pp. 161-172). Genève : Droz.

Berthoz, Alain (2009). La Simplexité. Paris : Odile Jacob.

Bremond, Claude (1998). L'exemplum médiéval est-il un genre littéraire ? In Jacques Berlioz \& Marie-Anne Polo de Beaulieu (dir.), Les Exempla médiévaux: Nouvelles perspectives (pp. 21-42). Paris : Champion.

CORRADI, FEDERICO (2013). L'illustration ésopique en Italie au XVI ${ }^{\mathrm{e}}$ siècle : les fabliers de Faërne et de Verdizzotti. Le Fablier. Revue des Amis de Jean de La Fontaine, $\mathrm{n}^{\mathrm{o}}$ 24, La Fontaine, la fable et l'image I, 73-82.

CuRTIUS, ERnSt (1986). La littérature européenne et le Moyen Âge latin. Trad. Jean Bréjoux. Paris : Agora.

Eichel-Lojkine, PATRicia (2013). Contes en réseaux. L'émergence des contes sur la scène littéraire européenne. Genève : Droz.

Flahault, François (2001). La Pensée des contes. Paris : Anthropos.

FoucAult, Michel (2008). L'Archéologie du savoir. Paris : Gallimard.

Heidmann, Ute (2011). Expérimentation générique et dialogisme intertextuel : Perrault, La Fontaine, Straparola, Basile. Féeries, $n^{\circ} 8, E$ Etudes sur le conte merveilleux (XVI $I^{e} X I X^{e}$ siècles), 45-69.

Heidmann, Ute et Adam Jean-Michel (2010). Textualité et intertextualité des contes. Perrault, Apulée, La Fontaine, Lhéritier. Paris : Garnier.

Jolles, Andre (1970). Formes simples. Trad. Antoine Marie Buguet. Paris : Seuil.

La Fontaine, JeAn DE (1990). Les amours de Psyché et de Cupidon. Éd. Françoise Charpentier. Paris : Flammarion.

LÜTHI, MAX (1986). The European Folktale. Form and Nature. Trad. John D. Niles. Bloomington \& Indianapolis : Indiana University Press.

Martin, Serge (1997). Les Contes à l'école. Paris : Bertrand-Lacoste.

PAUlme, Denise (1976). La Mère dévorante. Essai sur la morphologie des contes africains. Paris : Gallimard.

Perrault, Charles (1991). Contes. Éd. Gilbert Rouger. Paris : Garnier.

- (1692) Parallèle des Anciens et des Modernes, III, La Poésie Paris, Veuve Coignard. <http://reader.digitale-sammlungen.de/de/fs1/object/display/bsb10447865_00005.html>.

- (1989). Contes. Éd. Marc Soriano. Paris : Flammarion.

Roberts, WARren Everett (1994). The Tale of the Kind and Unkind Girls : AA-TT 480 and related titles. Detroit : Wayne State University Press. 
RóHeim, GÉZA (1992). Dame Holle : Dream and Folktale (Grimm No 24). In Géza Róheim, Fire in the Dragon and Other Psychoanalytic Essays on Folklore (pp. 181-192). Éd. Alan Dundes. Princeton : Princeton University Press.

Schmitt, Jean-Claude (dir.) (1985). Prêcher d'exemple. Récits de prédicateurs du Moyen-Âge. Paris : Stock.

Sermain, JeAn-Paul (2010a). Fables, contes, nouvelles. Liaisons poétiques. Féeries, 7, 9-19.

- (2010b). Ce que les contes doivent aux fées. Liaisons anthropologiques », Féeries, 7, 193-201.

SimOnSEn, Michele (1992). Perrault, Contes. Paris : PUF.

Straparola, Gianfrancesco (1585). Les nuictz facetieuses. Trad. Pierre de Larivey. Paris : Abel L'Angelier.

- (1857). Les Facétieuses nuits. Trad. Jean Louveau et Pierre de Larivey. Paris : P. Jannet, 2 vol. (Bibliothèque elzévirienne) (Gallica, en ligne : 〈http://gallica.bnf.fr/ark:/12148/bpt6k277945>; <http://gallica.bnf.fr/ark:/12148/bpt6k27795h)>.

- (1999). Les Nuits facétieuses. Trad. rev. par Joël Gayraud. Paris : J. Corti.

Thirard, Marie-Agnes (2004). Le renouveau à l'école à l'heure de la mondialisation. In J. Pierrot (dir.), Les métamorphoses du conte. Bruxelles : Peter Lang.

Tolan, John Victor (1993). Petrus Alfonsi and his medieval readers. Gainesville : University Press of Florida.

Velay-Vallantin, Catherine (1992). L'Histoire des contes. Paris : Fayard.

Viala, Alain (2008). La France galante. Paris : PUF.

- (2009). «Si Peau d'Âne m'était conté...» ou les frontières de la galanterie. Littératures classiques, 69, 79-88.

Wetzel, Hermann H. (1981). Éléments socio-historiques d'un genre littéraire : l'histoire de la nouvelle jusqu'à Cervantès. In Lionello Sozzi (dir.), La Nouvelle française de la Renaissance (pp. 41-80). Genève - Paris : Slatkine. 
